\title{
Certificación energética de edificios en España y sus implicaciones económicas
}

\author{
Spanish energy rating labelling of buildings and its cost \\ implications
}

$\underline{\text { M. J. Ruá }^{(*)}}{ }^{(\text {B. López-Mesa }}{ }^{(* *)}$

\section{RESUMEN}

Bajo la Directiva Europea sobre eficiencia energética de los edificios, los Estados Miembros deben desarrollar procedimientos para certificar energéticamente los edificios. Calener-VYP v1.0 es la única herramienta oficial española para certificar una elevada aptitud en eficiencia energética de edificios residenciales, por el método general. Tiene dos indicadores implementados, emisiones de $\mathrm{CO}_{2}$ y consumo de energía primaria. Sin embargo, en el proceso de otorgar la calificación energética, solamente se consideran las emisiones de $\mathrm{CO}_{2}$. Las implicaciones económicas de esto se estudian simulando viviendas con diferentes características constructivas e instalaciones en las doce zonas climáticas españolas y calculando sus costes. Los resultados muestran que una mejor calificación energética en Calener-VYP no implica necesariamente un menor coste de consumo energético. Por tanto, es necesario incluir el indicador consumo de energía primaria, ya que su ausencia significa que los costes energéticos no pueden ser usados como argumento para promover la eficiencia energética entre usuarios.

\section{$113-116$}

Palabras clave: Calener-VYP; certificación energética de edificios; emisiones de $\mathrm{CO}_{2}$ coste de consumo energético; coste de amortización.

\section{SUMMARY}

Under the European Energy Performance of Buildings Directive, the Member States must develop methods to certify energy efficiency of buildings. Calener-VYP v1.0 is the only Spanish official tool to certify a high energy efficiency performance of residential buildings, through the general option. It has two indicators implemented, $\mathrm{CO}_{2}$ emissions and primary energy consumption. However, in the process of energy rating a building, only the $\mathrm{CO}_{2}$ emissions indicator is considered. The economic consequences of this are studied, by means of simulating real properties with different construction assemblies and building services in the twelve Spanish climatic zones and calculating their costs. The results show that having a better energy rate in Calener VYP does not necessarily entail a lower consumption cost. Therefore, it is necessary to include the annual primary energy consumption indicator, because its absence involves that costs cannot be used as an argument when promoting energy efficiency among users.
Keywords: Calener-VYP; building efficiency rating, $\mathrm{CO}_{2}$ emissions; energy efficiency labelling; energy consumption cost; amortization cost.

\footnotetext{
(*) Universitat Jaume I. Castelló de la Plana (España).

(**) Universidad de Zaragoza (España).

Persona de contacto/Corresponding author: rua@uji.es. (M. J. Ruá).
}

Recibido/Received: 13 mar 2011 Aceptado/Accepted: 12 oct 2011 Publicado online/ Published online: 31 may 2012 


\section{INTRODUCCIÓN}

Reducir el consumo energético de los edificios es uno de los principales objetivos de la Unión Europea (UE) para alcanzar los compromisos adquiridos con su adhesión al Protocolo de Kioto, al mismo tiempo que se mejora el nivel de confort y se disminuyen las facturas energéticas de los ciudadanos (1).

En la UE y EEUU entre un 20 y $40 \%$ de la energía es consumida por los edificios, por encima de industria y transporte (2), por lo que el potencial del sector de la construcción en la reducción del consumo energético es evidente. Por esta razón, la UE aprobó la directiva 2002/91/EC (EPBD), modificada por la 2010/31/UE, cuyo fin es la promoción de mejoras costo-efectivas para mejorar la eficiencia energética de los edificios (3).

Las directivas EPBD 2002/91/EC y 2010/31/ UE tratan las necesidades energéticas de calefacción, refrigeración, agua caliente sanitaria (ACS) e iluminación en los edificios. Bajo esta normativa, los Estados Miembros deben aplicar requerimientos mínimos para calificar energéticamente edificios nuevos y existentes, así como procedimientos para certificar energéticamente dichos edificios. La Directiva establece principios básicos, dejando flexibilidad a los Estados Miembros para establecer mecanismos específicos, requisitos numéricos y formas de implementarlos, teniendo en cuenta sus condiciones locales (1).

En España, la EPBD fue parcialmente transpuesta por medio de tres reales decretos:

- Real Decreto que aprueba el Código Técnico de la Edificación (CTE), aprobado por Consejo de Ministros el 17 de Marzo de 2006 y publicado en el Boletín Oficial del Estado el 28 de Marzo de 2006. Uno de sus documentos básicos, el CTE-HE, recoge los requisitos del EPBD con respecto al ahorro de energía y al uso de energías renovables. Estos requisitos son de obligado cumplimiento en nueva edificación y reformas mayores en edificios existentes, desde el 17 de septiembre de 2006.

- Real Decreto sobre el Procedimiento Básico para la certificación de eficiencia energética de edificios de nueva construcción, aprobado por Consejo de Ministros el 17 de Enero de 2007 y publicado en el Boletín Oficial del Estado el 31 de Enero de 2007. La certificación de nuevos edificios es obligatoria para edificios cuya Licencia de Obras fuera concedida a partir del 31 de Octubre de 2007.

- Real Decreto que aprueba la revisión del Reglamento de Instalaciones Térmicas de los Edificios (RITE), aprobado por Consejo de Ministros el 20 de Julio de 2007 y publicado en el Boletín Oficial del Estado el 29 de Agosto de 2007. El RITE entró en vigor el 1 de Marzo de 2008.

La Directiva EPBD fue parcialmente transpuesta en España, ya que la certificación para edificios existentes no queda recogida en los reales decretos mencionados y está todavía en fase de aprobación administrativa.

En la mayor parte de Estados Miembros, la escala de calificación energética para representar la certificación de la eficiencia energética depende de dos tipos de indicadores: consumo anual de energía primaria, expresado en $\mathrm{kW} \cdot \mathrm{h}$ por $\mathrm{m}^{2}$ construido, y emisiones de dióxido de carbono, expresadas en $\mathrm{kg}$ $\mathrm{CO}_{2}$ por $\mathrm{m}^{2}$ construido $(1,4,5)$. En el caso español, ambos indicadores han sido incluidos en la metodología definida para determinar la calificación energética (6). Sin embargo, la herramienta oficial desarrollada para obtener la etiqueta de calificación energética en la opción general, Calener-VYP v1.0 (7) (en adelante Calener-VYP), pese a calcular ambos indicadores, solamente considera el indicador $\mathrm{kg} \mathrm{CO}_{2} / \mathrm{m}^{2}$ al certificar energéticamente el edificio. Esto se puede deducir de la etiqueta que genera el programa tras la simulación y del informe del Ministerio de Industria, Turismo y Comercio sobre condiciones de aceptación de procedimientos alternativos a Calener-VYP (8).

Además de Calener-VYP, existe la opción simplificada para el cálculo de la eficiencia energética. Esta se puede utilizar siempre que el edificio cumpla unos requisitos mínimos (menos del $60 \%$ de la superficie de huecos en fachadas y del $10 \%$ de lucernarios en cubiertas). Existen varias metodologías aprobadas para aplicar la opción simplificada (CE2, CES, CERMA, etc.). Sin embargo, Calener-VYP es la única herramienta oficial para la opción general, siendo obligatoria si se sobrepasan los mínimos indicados y representando la única vía para optar a las calificaciones mejores.

Mediante el uso de Calener-VYP se obtiene una etiqueta con la que se promociona la eficiencia energética, ya que compradores y usuarios disponen de información objetiva en relación con las características energéticas de los edificios, pudiendo seleccionar edificios más eficientes por criterios medioambientales y/o por el supuesto ahorro de costes de consumo energético propio de las mejores calificaciones energéticas. Por ello, pese a que la herramienta CalenerVYP ha sido concebida para la certificación energética de edificios, inherente a su existencia está el promocionar medidas costoefectivas que supongan un ahorro en las fac- 
turas energéticas. Por tanto, las herramientas de certificación deberían contemplar tanto el consumo energético como sus emisiones asociadas. Sin embargo, en Calener-VYP el consumo energético parece ser obviado a la hora de otorgar la calificación energética. Los objetivos de este artículo son verificar que efectivamente el indicador $\mathrm{kg} \mathrm{CO}_{2} / \mathrm{m}^{2}$ es el único que considera la herramienta y analizar cuáles son las consecuencias.

\section{METODOLOGÍA}

Este trabajo analiza la forma de otorgar la calificación energética del programa Calener VYP. Para ello, se trabaja sobre una vivienda tipo de una promoción de viviendas unifamiliares adosadas, utilizando un paquete de medidas que influyan en la eficiencia energética del edificio. Estas medidas comprenden algunos cambios respecto a la vivienda original que no afectan al diseño ni al uso de medidas pasivas, modificándose solamente algunos elementos constructivos y sistemas energéticos. Se analiza la influencia de dichos cambios en la calificación energética del edificio y, por otro lado, su coste económico y el coste o ahorro energético obtenido. Se pretende así, conocer la costo-efectividad de las medidas implementadas. Este estudio se desarrolla para cada zona climática en España y siguiendo el criterio de cambiar de calificación al menor coste de inversión posible.

Para llevar a cabo este estudio, IBERDROLA Inmobiliaria, promotora inmobiliaria dentro del grupo IBERDROLA, proporcionó la información de una de sus promociones consistente en 13 viviendas adosadas. Se seleccionó una vivienda en esquina, al presentar peores condiciones que las medianeras desde el punto de vista de la eficiencia energética. La fachada más larga está expuesta al exterior y a nivel de sótano posee una mayor superficie de muro en contacto con el terreno. De entre las seis viviendas en esquina, se seleccionó aquella orientada $25^{\circ} \mathrm{N}$, en la que la mayor superficie de fachada tiene orientación NW (Figura 1).

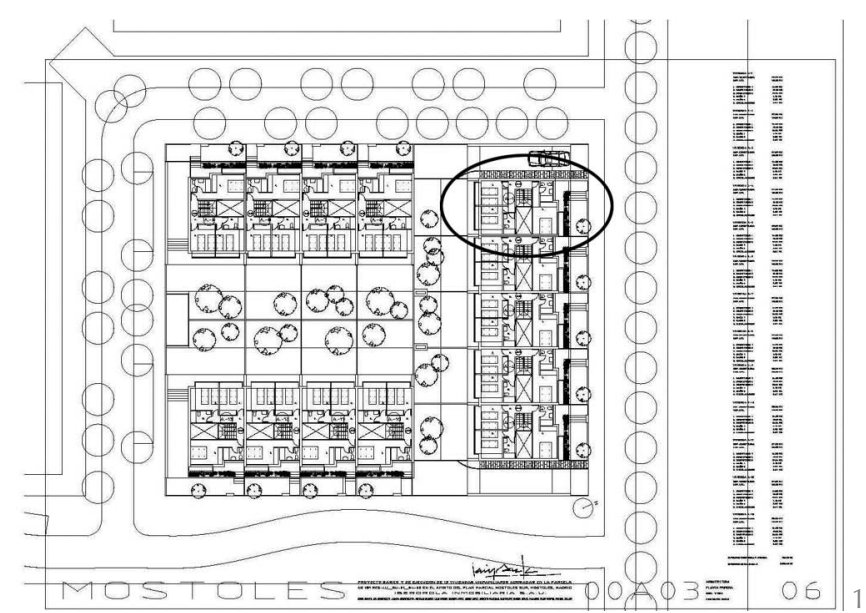

La totalidad de la promoción ocupa $2.332 \mathrm{~m}^{2}$ de terreno, $154-157 \mathrm{~m}^{2}$ por casa y zonas de uso común. Cada vivienda posee tres plantas. La planta sótano se destina a aparcamientos y cuartos de instalaciones. Todas las propiedades son accesibles desde el sótano, donde existe una entrada común para todos los propietarios (Figura 2), si bien cada vivienda posee su acceso individual (Figura 3).

Las plantas baja y primera (Figuras 2 y 3 ) se destinan a uso vivienda. La planta baja cuenta con $68,10 \mathrm{~m}^{2}$ de superficie distribuidos en recibidor, salón comedor, cocina y baño. La primera planta tiene $58,88 \mathrm{~m}^{2}$, ocupados por tres dormitorios y tres baños.

Las etapas seguidas en el estudio son:

1. Estudio de la escala de calificación para edificios residenciales en España, calculando los valores límite para cada nivel de eficiencia energética, de acuerdo al informe escrito por AICIA -Grupo de Termotecnia de la Escuela Superior de Ingenieros Industriales de la Universidad de Sevilla (6), que describe la metodología oficial para calificación energética, incluyendo los dos tipos de indicadores, consumo anual de energía primaria y emisiones anuales de $\mathrm{CO}_{2}$.
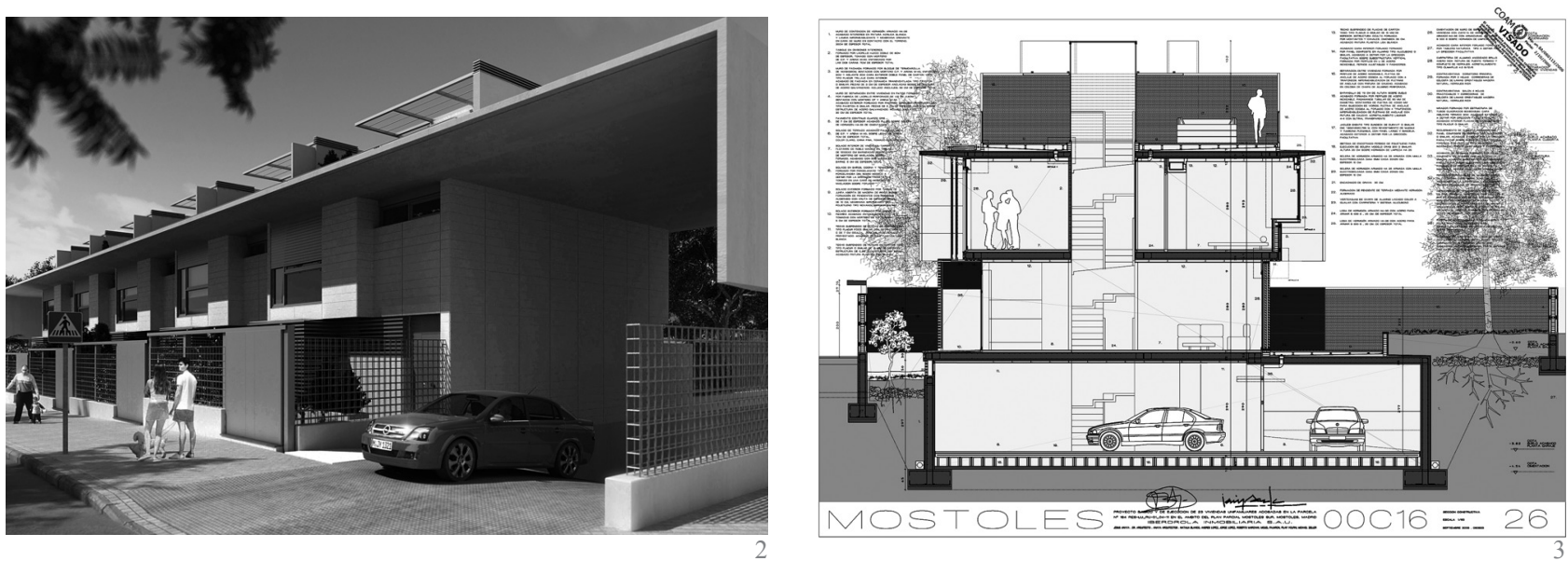
2. Simulación del comportamiento de la vivienda adosada en Calener-VYP para las diferentes zonas climáticas españolas. Se seleccionan inicialmente las soluciones de la envolvente térmica que permiten el cumplimiento de los requerimientos mínimos del CTE, sin excederlos, pero sí ajustando las soluciones a los formatos comerciales de los productos, teniendo en cuenta simultáneamente el menor coste de inversión posible. Como resultado se obtiene el nivel de eficiencia energética del edificio, así como el consumo anual de energía primaria y emisiones anuales de $\mathrm{CO}_{2}$.

3. Estudio de la reducción en consumo energético alcanzada, reemplazando una por una las soluciones iniciales por otras, tanto a nivel de soluciones constructivas como de instalaciones y el coste de inversión de dichos cambios.

4. Conocida la reducción en consumo energético para cada cambio y su coste de inversión, se analiza la combinación de cambios que posibilita el paso de una calificación energética a la siguiente, considerando el menor coste de inversión posible. Para cada combinación se obtienen el consumo de energía primaria y emisiones de $\mathrm{CO}_{2}$ anuales, por medio de Calener-VYP.

5. Se comprueba el uso del indicador $\mathrm{kg}$ $\mathrm{CO}_{2}$ por $\mathrm{m}^{2}$ como único utilizado en la herramienta, y se extraen conclusiones de acuerdo a las variaciones en el consumo de energía y los costes que se deriven de ellas.

6. Se calcula el coste de inversión, de amortización y de consumo energético para cada combinación de soluciones consideradas, para obtener cada clase energética, extrayéndose conclusiones sobre las implicaciones económicas del uso de indicadores en Calener-VYP, de acuerdo a los valores calculados para el caso de estudio.

\section{RESULTADOS}

\subsection{Escala de eficiencia energética en edificios residenciales en España}

La forma de establecer la escala de eficiencia energética utilizada en España se detalla en el informe Escala de calificación energética. Edificios de nueva construcción (6). De acuerdo a este documento, hay unos valores límite de los dos indicadores para las cinco clases energéticas que se utilizan en España en edificios de nueva construcción (de A a E). Estos dependen de la tipología constructiva, zona climática y municipio y de la contribución solar mínima para producción de ACS en dicho municipio. En esta escala, A se usa para las mejores calificaciones energéticas y $E$ para las peores (en edificios existentes también pueden utilizarse las letras F y G). En este caso se han analizado los valores límite de ambos indicadores, de acuerdo a (6) para el caso de viviendas unifamiliares. Los resultados aparecen en la Tabla 1.

Las condiciones climáticas en España se representan por una letra y un número. La letra va desde $A$ hasta $E$, representando $A$ las condiciones de invierno más benignas y E las más duras. El número está comprendido entre 1 y 4 , para las condiciones de verano más benignas y más duras, respectivamente. Hay un total de doce zonas climáticas en España, representadas en la primera columna de la Tabla 1, habiéndose elegido para cada zona una ciudad representativa. Se muestran los valores límite expresados en $\mathrm{kg} \mathrm{CO}_{2} / \mathrm{m}^{2}$. año en la primera fila y en $\mathrm{kW} \cdot \mathrm{h} / \mathrm{m}^{2} \cdot$ año en la segunda fila, para cada zona.

\subsection{Soluciones para cumplir con los requisitos del CTE al menor coste de inversión posible y niveles de eficiencia energética resultantes}

La Tabla 2 muestra las soluciones inicialmente adoptadas para la envolvente térmica e instalaciones del edificio para cumplir con los requerimientos mínimos del CTE al menor coste de inversión posible.

Como los requerimientos mínimos que establece el CTE difieren dependiendo de las diferentes zonas climáticas, se presentan tres soluciones iniciales que se muestran en rasgos generales en la Tabla 2, una común en las zonas climáticas A y B, otra en C, y una tercera para las zonas $\mathrm{D}$ y $\mathrm{E}$.

Al simular la vivienda en Calener-VYP 1.0, con las soluciones indicadas en la Tabla 2, se obtuvo la calificación D en las zonas A3, A4, B3, C3, C4, D3 y E1, y calificación E en los demás casos.

\subsection{Reducción de emisiones alcanzada al cambiar las soluciones constructivas e instalaciones de partida y costes asociados}

Desde las fases de diseño o de proyección del edificio se puede controlar en gran medida el consumo energético (9). Los elementos pasivos de diseño juegan un papel determinante a través de la adaptación climática del edificio al lugar en el que se ubica (10). Las estrategias para reducir las emisiones incluyen tanto elementos activos como pasivos:

- Estrategias para reducir las emisiones debidas a calefacción:

- Mejorar la orientación del edificio.

- Cambiar las soluciones de la envolvente térmica (muros Trombe, fachadas ventiladas, etc). 


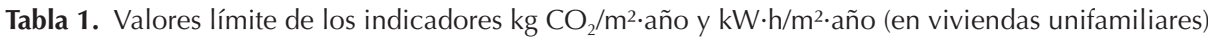

\begin{tabular}{|c|c|c|c|c|c|c|c|}
\hline \multirow{3}{*}{$\begin{array}{c}\text { Zona } \\
\text { climática }\end{array}$} & \multirow{3}{*}{ Ciudad } & \multirow{3}{*}{$\begin{array}{l}\text { Contribución } \\
\text { solar mínima }\end{array}$} & \multirow{2}{*}{\multicolumn{2}{|c|}{ Límites nivel Eficiencia Energética }} & \multicolumn{3}{|l|}{$\mathrm{kg} \mathrm{CO}_{2} / \mathrm{m}^{2}$.año } \\
\hline & & & & & $\mathrm{kW} \cdot \mathrm{h} / \mathrm{m}^{2} \cdot \mathrm{año}$ & & \\
\hline & & & A & B & C & $\mathrm{D}$ & $E$ \\
\hline \multirow{2}{*}{ A3 } & \multirow{2}{*}{ Málaga } & \multirow{2}{*}{$60 \%$} & $<4,6$ & $4,6-8,9$ & $8,9-14,9$ & $14,9-24$ & $>24$ \\
\hline & & & $<19,9$ & $19,9-38,2$ & $38,2-64,2$ & $64,2-103,2$ & $>103,2$ \\
\hline \multirow{2}{*}{ A4 } & \multirow{2}{*}{ Almería } & \multirow{2}{*}{$70 \%$} & $<4,4$ & $4,4-8,3$ & $8,3-14$ & $14-22,6$ & $>22,6$ \\
\hline & & & $<18,6$ & $18,6-35,7$ & $35,7-60,0$ & $60,0-96,4$ & $>96,4$ \\
\hline \multirow{2}{*}{ B3 } & \multirow{2}{*}{ Castellón } & \multirow{2}{*}{$60 \%$} & $<5,4$ & $5,4-10,4$ & $10,4-17,4$ & $17,4-28$ & $>28$ \\
\hline & & & $<23,6$ & $23,6-45,1$ & $45,1-75,9$ & $75,9-122,0$ & $>122,0$ \\
\hline \multirow{2}{*}{ B4 } & \multirow{2}{*}{ Sevilla } & \multirow{2}{*}{$70 \%$} & $<6,3$ & $6,3-11$ & $11-17,9$ & $17,9-28,1$ & $>28,1$ \\
\hline & & & $<26,9$ & $26,9-47,4$ & $47,4-76,6$ & $76,6-120,5$ & $>120,5$ \\
\hline \multirow{2}{*}{$\mathrm{C} 1$} & \multirow{2}{*}{ Santander } & \multirow{2}{*}{$30 \%$} & $<7,8$ & $7,8-12,7$ & $12,7-19,8$ & $19,8-30,4$ & $>30,4$ \\
\hline & & & $34,5-56,4$ & $56,4-87,7$ & $87,7-134,7$ & $>134,7$ & $34,5-56,4$ \\
\hline \multirow{2}{*}{$\mathrm{C} 2$} & \multirow{2}{*}{ Barcelona } & \multirow{2}{*}{$30 \%$} & $<7,9$ & $7,9-13$ & $13-20,2$ & $20,2-31$ & $>31$ \\
\hline & & & $<34,6$ & $34,6-56,6$ & $56,6-88,0$ & $88,0-135,2$ & $>135,2$ \\
\hline \multirow{2}{*}{ C3 } & \multirow{2}{*}{ Granada } & \multirow{2}{*}{$60 \%$} & $<8,2$ & $8,2-14$ & $14,4-23,2$ & $23,2-36,6$ & $>36,6$ \\
\hline & & & $<35,9$ & $35,9-63,3$ & $63,3-102,4$ & $102,4-161,1$ & $>161,1$ \\
\hline \multirow{2}{*}{ C4 } & \multirow{2}{*}{ Badajoz } & \multirow{2}{*}{$70 \%$} & $<7$ & $7-12,4$ & $12,4-20$ & $20-31,5$ & $>31,5$ \\
\hline & & & $<31,5$ & $31,5-55,5$ & $55,5-89,8$ & $89,8-141,3$ & $>141,3$ \\
\hline \multirow{2}{*}{ D1 } & \multirow{2}{*}{ Pamplona } & \multirow{2}{*}{$30 \%$} & $<13,2$ & $13,2-20,2$ & $20,2-30,2$ & $30,2-45,2$ & $>45,2$ \\
\hline & & & $<59,0$ & $59,0-90,2$ & $90,2-134,8$ & $134,8-201,7$ & $>201,7$ \\
\hline בס & L I & $50 \%$ & $<10,9$ & $10,9-17,8$ & $17,8-27,8$ & $27,8-42,6$ & $>42,6$ \\
\hline D2 & Logrono & $50 \%$ & $<46,2$ & $46,2-75,6$ & $75,6-117,6$ & $117,6-180,5$ & $>180,5$ \\
\hline & & & $<10$ & $10-16,4$ & $16,4-25,4$ & $25,4-39,1$ & $>39,1$ \\
\hline D3 & Madrid & $60 \%$ & $<44,2$ & $44,2-72,3$ & $72,3-112,5$ & $112,5-172,7$ & $>172,7$ \\
\hline E1 & & & $<16,9$ & $16,9-25,9$ & $25,9-38,7$ & $38,7-57,9$ & $>57,9$ \\
\hline EI & Burgos & $30 \%$ & $<75,8$ & $75,8-115,9$ & $115,9-173,2$ & $173,2-259,2$ & $>259,2$ \\
\hline
\end{tabular}

Tabla 2. Soluciones constructivas e instalaciones iniciales

\begin{tabular}{|c|c|c|c|}
\hline & Zonas climáticas A-B & Zonas climáticas C & Zonas climáticas D-E \\
\hline Calefacción & Termo eléctrico & Termo eléctrico & Termo eléctrico \\
\hline Aire acondicionado & Splits con bomba de calor & Splits con bomba de calor & Splits con bomba de calor \\
\hline $\begin{array}{l}\text { Mínima contribución solar } \\
\text { para ACS }\end{array}$ & Mínimos en CTE (tabla 1) & Mínimos en CTE (tabla 1) & Mínimos en CTE (tabla 1) \\
\hline $\begin{array}{l}\text { Material aislamiento en } \\
\text { envolvente (excepto fachada) }\end{array}$ & PUR $3 \mathrm{~cm}$ & PUR $3 \mathrm{~cm}$ & PUR $5 \mathrm{~cm}$ \\
\hline Fachada & $\begin{array}{l}\text { Doble hoja de ladrillo con } \\
\text { cámara y lana mineral } 5 \mathrm{~cm}\end{array}$ & $\begin{array}{l}\text { Doble hoja de ladrillo con } \\
\text { cámara y lana mineral } 5 \mathrm{~cm}\end{array}$ & $\begin{array}{l}\text { Doble hoja de ladrillo con } \\
\text { cámara y lana mineral } 5 \mathrm{~cm} \text { y } \\
\text { barrera de vapor }\end{array}$ \\
\hline Cubierta & Cubierta invertida & Cubierta invertida & Cubierta invertida \\
\hline Forjado & Hormigón armado & Hormigón armado & Hormigón armado \\
\hline Renovaciones aire & $0,6 \mathrm{~h}^{-1}$ & $0,6 \mathrm{~h}^{-1}$ & $0,6 \mathrm{~h}^{-1}$ \\
\hline Perfiles ventanas & $\begin{array}{l}\text { Perfil aluminio sin rotura } \\
\text { puente térmico }\end{array}$ & $\begin{array}{l}\text { Perfil aluminio con rotura } \\
\text { puente térmico }\end{array}$ & $\begin{array}{l}\text { Perfil aluminio con rotura } \\
\text { puente térmico }\end{array}$ \\
\hline Tipos vidrios & $4-6-6$ y $4-6-4$ & $4-6-6$ y $4-6-4$ & $6-12-4$ y $6-12-8$ \\
\hline
\end{tabular}

- Reducir las pérdidas de energía a través de los huecos e incrementar la ganancia de radiación solar (optimización de la forma y el tamaño de las ventanas, mejorar el valor de la transmitancia en los huecos).

- Incrementar el aislamiento (materiales con menor conductividad térmica, mayor espesor de los aislamientos).

- Reducir los puentes térmicos.

- Cambiar los sistemas de climatización (radiadores, suelo radiante).

- Cambiar la fuente de energía (gasóleo, gas natural, energía geotérmica).
- Estrategias para reducir las emisiones debidas a refrigeración:

- Mejorar la orientación del edificio.

- Cambiar las soluciones de la envolvente térmica (muros Trombe, fachadas ventiladas, etc).

- Incrementar las renovaciones de aire en verano.

- Reducir la radiación solar en verano optimizando la orientación y tamaño de los huecos, los sombreamientos, y empleando vidrios de control solar.

- Estrategias para reducir las emisiones debidas a la producción de ACS:

- Incrementar la contribución solar. 
No obstante, en este estudio, no se abordan todas las medidas pasivas porque se parte de un edificio existente. Los posibles nuevos diseños a analizar de la vivienda pueden ser infinitos al ser el diseño una actividad creativa. Por ello, se decidió no incluir en este estudio las estrategias que implicaban cambios en el diseño del edificio, como las mencionadas de optimización de la orientación del edificio y sus huecos, tamaño de los mismos y ventilación cruzada. Considerarlas y comparar las mejoras obtenidas con los cambios aquí considerados constituye un estudio de gran interés para el futuro. Otros estudios centrados en el análisis de la efectividad de las medidas y sus costes sí han realizado estas comparaciones, como el estudio PRECOST \& E (10).

Otras estrategias fueron descartadas al no poder calcularse con Calener-VYP, por no estar implementados en la herramienta los algoritmos necesarios. Es el caso de los muros Trombé, fachadas ventiladas, suelos radiantes, energía geotérmica o energía solar fotovoltaica. Por lo tanto, estas medidas no serán utilizadas, a excepción de la fachada ventilada para la que se utilizó una aproximación a su comportamiento, basada en la norma UNEEN ISO 6946:1997/A1:2005 (11, 12).

Los cambios utilizados inicialmente fueron:

- Caldera gasoil / Caldera estándar gas natural (GN) / Caldera baja temperatura GN / Caldera condensación GN: el cambio consiste en cambiar el termo eléctrico por Caldera gasoil / Caldera estándar gas natural (GN) / Caldera baja temperatura GN / Caldera condensación $\mathrm{GN}$, respectivamente.

- Caldera de biomasa: el cambio consiste en cambiar el combustible de GN a biomasa. La caldera de biomasa requiere considerar un silo de almacenamiento de pellets, incluido en la solución.

- Splits + radiadores: en la solución original calefacción y refrigeración se resuelve utilizando splits con bomba de calor. El cambio probado consiste en utilizar emisores tipo radiadores para calefacción con caldera de gas natural o biomasa, manteniendo los splits para refrigeración.

- Mínimo CTE +15\% y +30\%: estas soluciones consisten en incrementar la contribución solar del ACS de los mínimos que establece el CTE, en un 15 y $30 \%$, respectivamente.

- Lana mineral de $8 \mathrm{~cm}$ y $10 \mathrm{~cm}$ : el cambio consiste en incrementar el espesor de la capa de aislamiento de fachada, inicialmente de $5 \mathrm{~cm}$.

- PVC y perfiles de madera en ventanas: los perfiles de madera y PVC presentan menor transmitancia (U) que los metálicos de las soluciones iniciales.
- Aumento de la superficie de huecos sombreados mediante lamas de aluminio: originalmente la vivienda disponía de lamas solamente en las carpinterías recayentes a cocina y patio inglés. En las zonas A3, A4 y B4 es necesario utilizarlos en otros huecos para obtener la calificación A.

- 6-12-4 y 6-12-8: En las zonas climáticas A, B y C, la solución de vidrio inicial permitida por el CTE (4-6-6 y 4-6-4), puede ser incrementada a 6-12-4 y 6-12-8 (en D y $\mathrm{E}$ ya es originalmente esta última solución, al ser la mínima requerida por CTE).

- Vidrios bajo emisivos: todos los cristales se cambian a bajo emisivos, manteniendo el espesor original.

La Tabla 3 muestra las características técnicas más relevantes de interés en este análisis, para los materiales e instalaciones utilizados.

Como se observa en la Tabla 4, la medida más efectiva en cuanto a la reducción de emisiones es el uso de caldera de biomasa, ya que Calener-VYP considera que este tipo de calderas emite $0 \mathrm{~kg} \mathrm{CO}_{2}$ al ser considerada la biomasa combustible neutral respecto a la emisión de $\mathrm{CO}_{2}$. Esta consideración quiere decir que las emisiones se consideran nulas, no porque el uso de biomasa suponga emitir cero $\mathrm{kg}$ de $\mathrm{CO}_{2}$ sino porque las emisiones son compensadas por el $\mathrm{CO}_{2}$ que se absorbió por la materia vegetal de la que procede el combustible utilizado (13).

Como es lógico, algunas medidas para la reducción de emisiones son más eficientes dependiendo de la zona climática donde sean aplicadas. Por ejemplo, el uso de vidrios bajo emisivos y el uso de radiadores presentan una importante reducción en zonas con inviernos fríos, mientras que las calderas de gasóleo pueden ser interesantes en zonas de inviernos suaves.

Finalmente la Tabla 4 muestra que con el uso del resto de medidas se obtienen menores reducciones de las emisiones.

Se ha calculado el coste de inversión de cada vivienda en cada zona climática, considerando las diferencias de precios entre regiones y realizando un presupuesto detaIlado en cada configuración zona climáticacalificación energética, obteniendo los Presupuestos de Ejecución Material (PEM) (14). Para ello se ha utilizado la base de datos del generador de precios de Cype Ingenieros, S.A., con precios del año 2009-2010.

Las soluciones más económicas son las relacionadas con el aumento del espesor de la capa de aislamiento, seguida del uso de radiadores, incremento del espesor del vidrio y el uso de los diferentes tipos de calderas. 
Tabla 3. Características técnicas de materiales e instalaciones empleados

\begin{tabular}{|c|c|c|}
\hline \multicolumn{2}{|l|}{ Parámetros } & Características técnicas \\
\hline \multirow{6}{*}{$\begin{array}{l}\text { Calefacción } \\
\text { (caldera) }\end{array}$} & Termo eléctrico & Capacidad total $=2,6 \mathrm{~kW}$; Rendimiento nominal $=0,92$ \\
\hline & C. gasoil & Capacidad total $=30,2 \mathrm{~kW} ;$ Rendimiento nominal $=0,88$ \\
\hline & C. estándar GN & Capacidad total $=31,4 \mathrm{~kW} ;$ Rendimiento nominal $=0,92$ \\
\hline & C. baja temperatura GN & Capacidad total $=32,0 \mathrm{~kW} ;$ Rendimiento nominal $=0,94$ \\
\hline & C. condensación GN & Capacidad total $=31,4 \mathrm{~kW}$; Rendimiento nominal $=0,98$ \\
\hline & C. biomasa & Capacidad total $=23,2 \mathrm{~kW}$; Rendimiento nominal $=0,91$ \\
\hline Aire acondicionado & Splits con bomba de calor & $\begin{array}{l}\text { Unidades exteriores ( } 2 \text { modelos) } \\
\text { Capacidad total refrigeración nominal }=3,9-8,0 \mathrm{~kW} \\
\text { Consumo referencia nominal }=1,2-2,5 \mathrm{~kW} \\
\text { Capacidad calorífica nominal }=4,4-9,6 \mathrm{~kW} \\
\text { Consumo calefacción nominal }=1,2-2,4 \mathrm{~kW} \\
\text { Unidades interiores ( } 2 \text { modelos) } \\
\text { Capacidad total refrigeración nominal }=1,9-5,5 \mathrm{~kW} \\
\text { Capacidad total calefacción nominal }=1,9-5,5 \mathrm{~kW}\end{array}$ \\
\hline Energía solar ACS & Mínimo CTE & Ver tabla 1 \\
\hline \multirow{3}{*}{ Material aislamiento } & Lana mineral $5 \mathrm{~cm}$ & $\lambda=0,041 \mathrm{~W} / \mathrm{m} \cdot \mathrm{K}$ \\
\hline & Lana mineral $8 \mathrm{~cm}$ & $\lambda=0,041 \mathrm{~W} / \mathrm{m} \cdot \mathrm{K}$ \\
\hline & Lana mineral $10 \mathrm{~cm}$ & $\lambda=0,041 \mathrm{~W} / \mathrm{m} \cdot \mathrm{K}$ \\
\hline \multirow{4}{*}{ Perfiles ventanas } & $\begin{array}{l}\text { Metálico sin rotura } \\
\text { de puente térmico }\end{array}$ & $\mathrm{U}=5,7 \mathrm{~W} / \mathrm{m}^{2} \cdot \mathrm{K}$ \\
\hline & $\begin{array}{l}\text { Metálico con rotura } \\
\text { de puente térmico }>12 \mathrm{~mm}\end{array}$ & $\mathrm{U}=3,2 \mathrm{~W} / \mathrm{m}^{2} \cdot \mathrm{K}$ \\
\hline & PVC & $\mathrm{U}=1,8 \mathrm{~W} / \mathrm{m}^{2} \cdot \mathrm{K}$ \\
\hline & Madera & $\mathrm{U}=2,0 \mathrm{~W} / \mathrm{m}^{2} \cdot \mathrm{K}$ \\
\hline \multirow{3}{*}{$\begin{array}{l}\text { U ventanas } \\
\text { (cálculos programa } \\
\text { Calumen de Saint Gobain) }\end{array}$} & $\begin{array}{l}4-6-4 \\
4-6-6\end{array}$ & $\mathrm{U}=3,3 \mathrm{~W} / \mathrm{m}^{2} \cdot \mathrm{K} ;$ factor solar 0,75 \\
\hline & $\begin{array}{l}6-12-4 \\
6-12-8\end{array}$ & $\mathrm{U}=2,8 \mathrm{~W} / \mathrm{m}^{2} \cdot \mathrm{K} ;$ factor solar 0,73 \\
\hline & Vidrio bajo emisivo & $\mathrm{U}=1,6 \mathrm{~W} / \mathrm{m}^{2} \cdot \mathrm{K} ;$ factor solar 0,42 \\
\hline
\end{tabular}

Tabla 4. Porcentaje de reducción de emisiones alcanzada cambiando la solución original

\begin{tabular}{|c|c|c|c|c|c|c|c|c|c|c|c|c|c|}
\hline \multirow{2}{*}{ Parámetros } & \multirow{2}{*}{$\begin{array}{l}\text { De menor } \\
\text { a mayor coste }\end{array}$} & & & & & & & & & & & & \\
\hline & & A3 & A4 & B3 & B4 & $\mathrm{C} 1$ & $\mathrm{C} 2$ & $\mathrm{C} 3$ & $\mathrm{C} 4$ & D1 & D2 & D3 & E1 \\
\hline \multicolumn{2}{|c|}{ Soluciones iniciales (Tabla 2) } & 100 & 100 & 100 & 100 & 100 & 100 & 100 & 100 & 100 & 100 & 100 & 100 \\
\hline \multirow{5}{*}{$\begin{array}{l}\text { Calefacción } \\
\text { (caldera) }\end{array}$} & C. gasoil & 85,3 & 80,9 & 88,4 & 84,6 & 86,1 & 91,9 & 90,7 & 86,7 & 94,7 & 93,8 & 98,7 & 95,8 \\
\hline & C. estándar GN & 78 & 74,6 & 82,6 & 79,4 & 77,7 & 83,1 & 85,9 & 82,4 & 88,5 & 88,6 & 86,6 & 88,7 \\
\hline & C. baja temperatura GN & 78 & 74,6 & 82,1 & 79,4 & 77,3 & 82,6 & 85,9 & 82 & 88,2 & 88,2 & 86,6 & 88,5 \\
\hline & C. condensación GN & 76,8 & 74 & 81,7 & 79 & 76,5 & 82,2 & 85,2 & 81,6 & 87,6 & 87,9 & 85,9 & 88,0 \\
\hline & C. biomasa & 65 & 67,6 & 69,6 & 68,7 & 67,7 & 64,4 & 75,9 & 72,9 & 83,1 & 77,1 & 77,1 & 77,7 \\
\hline Aire acondicionado & Splits + radiadores & 90,6 & 96,9 & 74,6 & 118,8 & 92,3 & 94,9 & 92,4 & 104,3 & 88,6 & 90 & 89,1 & 86,2 \\
\hline \multirow{2}{*}{ Energía solar ACS } & Mínimo CTE + 15\% & 94,9 & 94,6 & 95,7 & 95,9 & 95,9 & 96,4 & 97,2 & 96,2 & 97,5 & 97 & 97,4 & 99,6 \\
\hline & Mínimo CTE + 30\% & 89,9 & 89,1 & 91,9 & 91,8 & 91,8 & 92,3 & 94 & 92,9 & 94,9 & 94,1 & 94,3 & 94,9 \\
\hline \multirow{2}{*}{$\begin{array}{l}\text { Material } \\
\text { aislamiento }\end{array}$} & *Lana mineral $8 \mathrm{~cm}$ & 95,7 & 96,1 & 94,1 & 95,9 & 94,4 & 94,4 & 93,6 & 95,2 & 98,1 & 98,2 & 98,1 & 96,1 \\
\hline & *Lana mineral $10 \mathrm{~cm}$ & - & - & - & - & - & - & - & - & 96,8 & 96,7 & 96,2 & 94,5 \\
\hline \multirow{2}{*}{ Perfiles ventanas } & PVC & 96,4 & 96,9 & 93,5 & 96,5 & 93,3 & 95,9 & 92,8 & 97,1 & 93,3 & 92,3 & 92,1 & 91,1 \\
\hline & Madera & 97,1 & 96,9 & 94,6 & 96,5 & 94,4 & 94,9 & 93,6 & 95,7 & 94,3 & 93 & 93,2 & 92,0 \\
\hline \multirow{2}{*}{$U$ ventanas } & 6-12-4 and 6-12-8 & 94,5 & 94,9 & 92 & 93,4 & 96,5 & 92,5 & 92,9 & 94,2 & - & - & - & - \\
\hline & Vidrio bajo emisivo & 90,4 & 91,2 & 84,6 & 89 & 89,6 & 85,8 & 84,7 & 88,8 & 85,4 & 86,1 & 85,2 & 85,5 \\
\hline
\end{tabular}

Sin embargo, el cambio de los tipos de perfiles en carpinterías exteriores y la caldera de biomasa, suponen los mayores costes de inversión.

El coste de incrementar los porcentajes de contribución solar para producción de ACS, ha resultado nulo en la mayor parte de los casos, dado que los formatos comerciales utilizados alcanzaban porcentajes muy por encima de los mínimos, incluso por encima del 15 y el $30 \%$ considerado (excepto en un caso).

Finalmente, la Tabla 5 muestra el ratio conseguido de reducción de emisiones por euro de inversión en cada cambio.

De la Tabla 5 se deduce que el mayor ratio se consigue con el incremento del espesor 
Tabla 5. Porcentaje de reducción emisiones por euro de coste de inversión por cada cambio de la Tabla 3

\begin{tabular}{|l|c|c|c|c|c|c|c|c|c|c|c|c|}
\hline Zona climática & A3 & A4 & B3 & B4 & C1 & C2 & C3 & C4 & D1 & D2 & D3 & E1 \\
\hline C. gasoil & 0,042 & 0,040 & 0,043 & 0,042 & 0,042 & 0,044 & 0,046 & 0,044 & 0,046 & 0,046 & 0,047 & 0,048 \\
\hline C. estándar GN & 0,027 & 0,026 & 0,028 & 0,027 & 0,026 & 0,028 & 0,030 & 0,028 & 0,030 & 0,030 & 0,029 & 0,030 \\
\hline C. baja temperatura GN & 0,052 & 0,049 & 0,054 & 0,052 & 0,051 & 0,054 & 0,057 & 0,055 & 0,058 & 0,058 & 0,057 & 0,059 \\
\hline C. condensación GN & 0,022 & 0,021 & 0,023 & 0,023 & 0,022 & 0,023 & 0,025 & 0,024 & 0,025 & 0,025 & 0,024 & 0,025 \\
\hline C. biomasa & 0,006 & 0,007 & 0,007 & 0,007 & 0,007 & 0,006 & 0,008 & 0,007 & 0,008 & 0,008 & 0,008 & 0,008 \\
\hline Splits + radiadores & 0,084 & 0,078 & 0,058 & 0,097 & 0,081 & 0,068 & 0,061 & 0,069 & 0,050 & 0,063 & 0,056 & 0,085 \\
\hline Lana mineral 8 cm & 0,396 & 0,412 & 0,393 & 0,443 & 0,381 & 0,363 & 0,412 & 0,428 & 0,399 & 0,400 & 0,377 & 0,417 \\
\hline Lana mineral 10 cm & - & - & - & - & - & - & - & - & 0,167 & 0,167 & 0,157 & 0,174 \\
\hline Madera & 0,011 & 0,010 & 0,010 & 0,010 & 0,015 & 0,017 & 0,013 & 0,013 & 0,015 & 0,015 & 0,017 & 0,013 \\
\hline 6-12-4 and 6-12-8 & 0,064 & 0,064 & 0,062 & 0,063 & 0,065 & 0,063 & 0,063 & 0,059 & - & - & - & - \\
\hline Vidrio bajo emisivo & 0,032 & 0,032 & 0,030 & 0,031 & 0,030 & 0,029 & 0,028 & 0,031 & 0,029 & 0,029 & 0,029 & 0,029 \\
\hline
\end{tabular}

de la capa de aislamiento en la envolvente del edificio. La diferencia con el resto de medidas es significativa.

Las siguientes dos medidas más efectivas serían el uso de radiadores en calefacción y la mejora del vidrio, seguidas del uso de calderas y vidrios bajo emisivos. De entre las calderas, la de baja temperatura son más convenientes, si tenemos en cuenta emisiones y coste de inversión. Debido a su alto coste de inversión, las medidas que presentan peor ratio son la carpintería de madera y la caldera de biomasa.

\subsection{Combinación de cambios de la solución inicial, para la mejora de la calificación energética al menor coste de inversión posible}

La simulación con las soluciones iniciales obtenían la calificación D ó E, según zona climática, como se ha visto en el apartado 3.2. La Tabla 6 muestra los cambios finalmente realizados desde la solución inicial para conseguir calificaciones energéticas mejores, considerando que los cambios se realizan al menor coste de inversión posible. De las medidas manejadas, no se vuelve a utilizar la carpintería de PVC al obtener similares resultados a los de carpintería de madera. Por otro lado, se incluyen cristales de control solar y se aumenta el porcentaje de lamas para sombreamiento de huecos para poder alcanzar las calificaciones A en zonas con elevadas demandas en refrigeración. Las celdas en gris representan los cambios. Por ejemplo, en la zona B4, para obtener una calificación energética D, al menor coste de inversión posible, se incrementó el espesor de la capa de aislamiento de 5 a $8 \mathrm{~cm}$. En gris oscuro se representa los cambios que en ocasiones fueron necesarios hacer para obtener una calificación $B$, pero que resultaron más costosos que los cambios para llegar a la calificación A. Esta anomalía está relacionada con la consideración que Calener-VYP hace cuando el combustible es biomasa. Así, por ejemplo, en la zona E1, el uso de caldera de biomasa permite obtener directamente una calificación A. Por tanto, para alcanzar la calificación B no se puede utilizar esta medida, sino una combinación del resto de medidas, que resulta más costosa en su conjunto que el uso de la caldera de biomasa por sí sola. Por último, se representan con un aspa las combinaciones en las que ni siquiera la combinación de medidas conseguía alcanzar la calificación energética B.

A continuación se explican los cambios necesarios para mejorar la calificación energética, para la vivienda estudiada y las medidas manejadas:

- Cambio de calificación energética E a D: En las zonas climáticas B4, C1, C2, D1, D2 se alcanzaba la calificación E con la solución inicial. Incrementar la capa de aislamiento en espesor fue suficiente para alcanzar la calificación D en las zonas B4, D1 y D2, pero no en C1 y C2. En estos dos últimos casos, el uso de caldera de gas natural con radiadores resultó la solución más económica para alcanzar la calificación deseada.

- Cambio de calificación energética D a C: Se requiere el cambio a caldera de gas natural en todas las zonas. También el incremento del espesor de los vidrios, donde inicialmente se utilizaban vidrios 6-4-6 y 6-4-4. Asimismo es necesario el uso de carpintería de madera, a pesar de que los perfiles de madera son soluciones costosas, excepto en las zonas climáticas C3, C4, D1, D2.

- Cambio de calificación energética C a B: Esta calificación sólo se puede alcanzar utilizando caldera de biomasa en las zonas climáticas A3, A4, B3 y B4. En el resto de zonas, donde los requerimientos de refrigeración no son tan elevados, el uso de calderas de biomasa implica directamente una calificación A, como se ha explicado previamente. Para obtener la calificación B en las zonas climáticas C1, C2, D1, D2 
y E1 es necesario emplear la combinación de otras soluciones, obteniéndose en las zonas C1, C2 y D1 costes de inversión más elevados en $B$ que en $A$. En las zonas C3, C4 y D3 no fue posible alcanzar la calificación B con las soluciones consideradas en este trabajo. Posiblemente un diseño pasivo más apropiado a estas zonas habría resuelto esta cuestión, aunque esto no es objeto de este estudio.

- Cambio de calificación energética B a A: En todos los casos, la calificación A sólo se obtiene mediante el uso de caldera de biomasa. Además en las zonas más cálidas, A3, A4, B3 y B4, se hace necesario introducir otras medidas, no consideradas en un principio, para controlar las altas demandas de refrigeración. Se emplean vidrios de control solar y en las zonas A3, A4 y B4, se incrementa la superficie de lamas en ventanas como elemento de sombreamiento.

\subsection{Comprobación de si el indicador $\mathrm{kg} \mathrm{CO} / \mathrm{m}^{2} \cdot$ año es el único implementado o no para obtener la calificación energética en Calener VYP}

La Tabla 7 muestra los resultados de las emisiones anuales de $\mathrm{CO}_{2}$ y el consumo anual de energía primaria, obtenidos con Calener VYP, con las diferentes combinaciones de medidas utilizadas, para alcanzar las distintas calificaciones energéticas en la vivienda estudiada.

Comparando las Tablas 1 y 7 se observa que todos los valores de emisiones anuales de $\mathrm{CO}_{2}$ se encuentran dentro de los límites que establece la metodología (6). Sin embargo, la situación es distinta si se tiene en cuenta el indicador consumo anual de energía primaria, excediéndose en muchos casos los límites establecidos en (6). Las celdas sombreadas en gris señalan aquellos valores de consumo anual de energía primaria que exceden los límites establecidos por la metodología (Tabla 1), mientras que las cifras subrayadas indican los valores que no llegan a dichos límites.

Asimismo se puede observar en la Tabla 7, que el indicador $\mathrm{kW} \cdot \mathrm{h} / \mathrm{m}^{2} \cdot$ año no es menor cuanto mejor es la calificación energética, mientras que esto sí se cumple para el indicador $\mathrm{kg} \mathrm{CO}_{2} / \mathrm{m}^{2} \cdot$ año.

Por lo tanto, se puede concluir que el indicador emisiones anuales de $\mathrm{CO}_{2}$, en $\mathrm{kg}$ $\mathrm{CO}_{2} / \mathrm{m}^{2} \cdot$ año, es efectivamente el único utilizado en Calener-VYP v.1.0 a la hora de otorgar la etiqueta energética. En el próximo apartado se comentarán las consecuencias de este hecho.
Tabla 6. Cambios de la solución inicial para mejorar la eficiencia energética

\begin{tabular}{|c|c|c|c|c|c|c|c|c|c|c|c|c|c|}
\hline & & A3 & A4 & B3 & B4 & C1 & $\mathrm{C} 2$ & $\mathrm{C} 3$ & $\mathrm{C} 4$ & D1 & D2 & D3 & E1 \\
\hline \multirow{11}{*}{$\mathrm{D}$} & Carpintería de madera & & & & & & & & & & & & \\
\hline & Gas natural + radiadores & & & & & & & & & & & & \\
\hline & Glass 6-12-4; 6-12-8 & & & & & & & & & & & & \\
\hline & Vidrio control solar & & & & & & & & & & & & \\
\hline & Vidrio bajo emisivo & & & & & & & & & & & & \\
\hline & Incremento \% lamas & & & & & & & & & & & & \\
\hline & Lana mineral $8 \mathrm{~cm}$ & & & & & & & & & & & & \\
\hline & Lana mineral $10 \mathrm{~cm}$ & & & & & & & & & & & & \\
\hline & Fachada ventilada & & & & & & & & & & & & \\
\hline & Incremento \% solar ACS & & & & & & & & & & & & \\
\hline & Caldera biomasa+silo & & & & & & & & & & & & \\
\hline \multirow{11}{*}{ C } & Carpintería de madera & & & & & & & & & & & & \\
\hline & Gas natural + radiadores & & & & & & & & & & & & \\
\hline & Glass 6-12-4; 6-12-8 & & & & & & & & & & & & \\
\hline & Vidrio control solar & & & & & & & & & & & & \\
\hline & Vidrio bajo emisivo & & & & & & & & & & & & \\
\hline & Incremento \% lamas & & & & & & & & & & & & \\
\hline & Lana mineral $8 \mathrm{~cm}$ & & & & & & & & & & & & \\
\hline & Lana mineral $10 \mathrm{~cm}$ & & & & & & & & & & & & \\
\hline & Fachada ventilada & & & & & & & & & & & & \\
\hline & Incremento \% solar ACS & & & & & & & & & & & & \\
\hline & Caldera biomasa+silo & & & & & & & & & & & & \\
\hline \multirow{11}{*}{ B } & Carpintería de madera & & & & & & & & & & & & \\
\hline & Gas natural + radiadores & & & & & & & & & & & & \\
\hline & Glass $6-12-4 ; 6-12-8$ & & & & & & & & & & & & \\
\hline & Vidrio control solar & & & & & & & & & & & & \\
\hline & Vidrio bajo emisivo & & & & & & & & & & & & \\
\hline & Incremento \% lamas & & & & & & & & & & & & \\
\hline & Lana mineral $8 \mathrm{~cm}$ & & & & & & & & & & & & \\
\hline & Lana mineral $10 \mathrm{~cm}$ & & & & & & & & & & & & \\
\hline & Fachada ventilada & & & & & & & & & & & & \\
\hline & Incremento \% solar ACS & & & & & & & & & & & & \\
\hline & Caldera biomasa+silo & & & & & & & & & & & & \\
\hline \multirow{11}{*}{ A } & Carpintería de madera & & & & & & & & & & & & \\
\hline & Gas natural + radiadores & & & & & & & & & & & & \\
\hline & Glass 6-12-4; 6-12-8 & & & & & & & & & & & & \\
\hline & Vidrio control solar & & & & & & & & & & & & \\
\hline & Vidrio bajo emisivo & & & & & & & & & & & & \\
\hline & Incremento \% lamas & & & & & & & & & & & & \\
\hline & Lana mineral $8 \mathrm{~cm}$ & & & & & & & & & & & & \\
\hline & Lana mineral $10 \mathrm{~cm}$ & & & & & & & & & & & & \\
\hline & Fachada ventilada & & & & & & & & & & & & \\
\hline & Incremento \% solar ACS & & & & & & & & & & & & \\
\hline & Caldera biomasa+silo & & & & & & & & & & & & \\
\hline
\end{tabular}

3.6 Coste de inversión, amortización y consumo para las distintas combinaciones utilizadas en la obtención de las calificaciones energéticas

La Tabla 8 recoge el coste de amortización de la vivienda, calculada a partir de los costes de inversión. En el coste de inversión se ha tenido en cuenta los gastos generales y el beneficio industrial de la construcción (Presupuesto de Ejecución Contrata, suponiendo un incremento del 19\% sobre el Presupuesto de Ejecución Material). Para el cálculo de la amortización se ha considerado una vida útil del edificio de 100 años, cuando se trata de 
Tabla 7. Resultados de emisiones anuales de gases efecto invernadero en $\mathrm{kg} \mathrm{CO}_{2} \mathrm{~m}^{2}$.año para la vivienda adosada estudiada (celdas sombreadas: valores de consumo anual de energía primaria por encima de los límites; cifras subrayadas: valores que no llegan a los límites)

\begin{tabular}{|c|c|c|c|c|c|c|c|}
\hline \multirow{3}{*}{ Zona climática } & \multirow{3}{*}{ Ciudad } & \multirow{3}{*}{ Contribución solar mínima } & \multirow{2}{*}{\multicolumn{3}{|c|}{$\begin{array}{c}\text { Resultados de los indicadores para cada } \\
\text { calificación energética }\end{array}$}} & \multirow{2}{*}{\multicolumn{2}{|c|}{$\begin{array}{l}\mathrm{kg} \mathrm{CO} / \mathrm{m}^{2} \cdot \text { año } \\
\mathrm{kW} \cdot \mathrm{h} / \mathrm{m}^{2} \cdot \text { año }\end{array}$}} \\
\hline & & & & & & & \\
\hline & & & $A$ & B & $\mathrm{C}$ & $\mathrm{D}$ & $E$ \\
\hline \multirow{2}{*}{ A3 } & \multirow{2}{*}{ Málaga } & \multirow{2}{*}{$60 \%$} & 4,3 & 7,0 & 14,6 & 23,3 & - \\
\hline & & & 58,0 & 58,2 & 65,7 & 93,6 & - \\
\hline \multirow[b]{2}{*}{ A4 } & \multirow{2}{*}{ Almería } & \multirow[b]{2}{*}{$70 \%$} & 4,3 & 7,9 & 14,0 & 22,5 & - \\
\hline & & & 71,4 & 65,3 & 62,2 & 89,9 & - \\
\hline \multirow{2}{*}{ B3 } & \multirow{2}{*}{ Castellón } & \multirow{2}{*}{$60 \%$} & 5,4 & 6,0 & 16,7 & 27,5 & - \\
\hline & & & 80,3 & 80,3 & 76,9 & 110,4 & - \\
\hline \multirow{2}{*}{ B4 } & \multirow{2}{*}{ Sevilla } & \multirow{2}{*}{$70 \%$} & 6,2 & 10,0 & 17,5 & 27,7 & 28,2 \\
\hline & & & 107,4 & 79,5 & 77,1 & 111,2 & 112,9 \\
\hline \multirow{2}{*}{$\mathrm{C} 1$} & \multirow{2}{*}{ Santander } & \multirow{2}{*}{$30 \%$} & 2,4 & 12,7 & 19,6 & 30,3 & 33,5 \\
\hline & & & 71,9 & 60,3 & 95,6 & 109,0 & 134,4 \\
\hline \multirow{2}{*}{$\mathrm{C} 2$} & \multirow{2}{*}{ Barcelona } & \multirow{2}{*}{$30 \%$} & 7,0 & - & 22,9 & 36,2 & $\overline{-}$ \\
\hline & & & 100,3 & 58,4 & 95,3 & 105,9 & 129,9 \\
\hline \multirow{2}{*}{ C3 } & \multirow{2}{*}{ Granada } & \multirow{2}{*}{$60 \%$} & 7,0 & - & 22,9 & 36,2 & - \\
\hline & & & 113,6 & - & 107,1 & 144,6 & - \\
\hline \multirow{2}{*}{$\mathrm{C} 4$} & \multirow{2}{*}{ Badajoz } & \multirow{2}{*}{$70 \%$} & 6,5 & - & 20,0 & 31,1 & - \\
\hline & & & 73,8 & - & 91,8 & 124,7 & - \\
\hline \multirow{2}{*}{ D1 } & \multirow{2}{*}{ Pamplona } & \multirow{2}{*}{$30 \%$} & 3,1 & 19,4 & 28,6 & 44,9 & 46,0 \\
\hline & & & 151,2 & 93,9 & 139,0 & 180,0 & 184,4 \\
\hline & & & 4,5 & 17,8 & 27,7 & 42,6 & 43,6 \\
\hline D2 & Logrono & $50 \%$ & 136,8 & 84,9 & 133,6 & 171,1 & 174,8 \\
\hline & & $60 \%$ & 5,9 & - & 25,2 & 37,6 & - \\
\hline D3 & Madrıd & $60 \%$ & 125,7 & - & 119,5 & 150,8 & - \\
\hline 51 & & $20 \%$ & 2,0 & 24,7 & 32,1 & 51,8 & - \\
\hline ET & Burgos & $30 \%$ & 167,2 & 121,2 & 157,5 & 207,7 & - \\
\hline
\end{tabular}

Tabla 8. Amortización y coste anual de consumo de la vivienda, con cada combinación de medidas (en negrita: casos en a mejor calificación energética, los costes de consumo no son menores)

\begin{tabular}{|c|c|c|c|c|c|c|c|c|c|}
\hline Zona & $\begin{array}{l}\text { Eficiencia } \\
\text { energética }\end{array}$ & $\begin{array}{c}\text { Amortización } \\
(€ / \text { año })\end{array}$ & $\begin{array}{c}\text { Coste consumo } \\
(€ / a \tilde{a} 0)\end{array}$ & $\begin{array}{c}\text { Coste total } \\
(€ / \text { año })\end{array}$ & Zona & $\begin{array}{c}\text { Eficiencia } \\
\text { energética }\end{array}$ & $\begin{array}{c}\text { Amortización } \\
(€ / a n ̃ o)\end{array}$ & $\begin{array}{c}\text { Coste consumo } \\
(€ / a n ̃ o)\end{array}$ & $\begin{array}{l}\text { Coste total } \\
(€ / \text { año })\end{array}$ \\
\hline \multirow{4}{*}{ A3 } & $\mathrm{D}$ & $2.801,37$ & 627,90 & $3.429,27$ & \multirow{4}{*}{$\mathrm{C} 3$} & $\mathrm{D}$ & $2.714,30$ & 898,41 & $3.612,71$ \\
\hline & C & $3.182,84$ & 531,36 & $3.714,20$ & & C & $3.028,14$ & 724,22 & $3.752,36$ \\
\hline & $\mathrm{B}$ & $3.571,66$ & 485,13 & $4.056,79$ & & & & & \\
\hline & $A$ & $3.851,47$ & 431,27 & $4.282,74$ & & A & $3.617,31$ & 716,72 & $4.334,03$ \\
\hline \multirow{4}{*}{ A4 } & $\mathrm{D}$ & $2.823,41$ & 608,09 & $3.431,50$ & \multirow{4}{*}{$\mathrm{C} 4$} & $\mathrm{D}$ & $2.687,08$ & 792,96 & $3.480,04$ \\
\hline & $\mathrm{C}$ & $3.206,85$ & 516,91 & $3.723,76$ & & $\mathrm{C}$ & $3.005,58$ & 654,99 & $3.660,57$ \\
\hline & $B$ & $3.685,89$ & 471,06 & $4.156,95$ & & & & & \\
\hline & $A$ & $3.852,34$ & 499,58 & $4.351,92$ & & A & $3.127,90$ & 513,45 & $3.641,35$ \\
\hline \multirow{5}{*}{ B3 } & & & & & \multirow{5}{*}{ D1 } & $E$ & $2.491,32$ & 1109,29 & $3.600,61$ \\
\hline & $\mathrm{D}$ & $2.843,24$ & 716,72 & $3.559,96$ & & $\mathrm{D}$ & $2.492,97$ & 1085,75 & $3.578,72$ \\
\hline & $\mathrm{C}$ & $3.225,65$ & 580,87 & $3.806,52$ & & $\mathrm{C}$ & $3.238,28$ & 863,15 & $4.101,43$ \\
\hline & B & $3.734,16$ & 546,07 & $4.280,23$ & & B & $3.353,76$ & 651,17 & $4.004,93$ \\
\hline & A & $3.844,51$ & 546,02 & $4.390,53$ & & A & $3.813,86$ & 905,85 & $4.719,71$ \\
\hline \multirow{5}{*}{ B4 } & $E$ & $2.845,21$ & 730,06 & $3.575,27$ & \multirow{5}{*}{ D2 } & $E$ & $2.763,66$ & 1058,33 & $3.821,99$ \\
\hline & $\mathrm{D}$ & $2.847,28$ & 721,13 & $3.568,41$ & & $\mathrm{D}$ & $2.962,76$ & 1038,65 & $4.001,41$ \\
\hline & $\mathrm{C}$ & $3.242,56$ & 591,92 & $3.834,48$ & & $\mathrm{C}$ & $3.230,99$ & 841,82 & $4.072,81$ \\
\hline & B & $3.742,33$ & 545,16 & $4.287,49$ & & B & $3.351,58$ & 612,47 & $3.964,05$ \\
\hline & A & $3.860,64$ & 684,49 & $4.545,13$ & & A & $3.700,65$ & 833,48 & $4.534,13$ \\
\hline \multirow{5}{*}{ C1 } & $E$ & $2.954,79$ & 844,23 & $3.799,02$ & \multirow{5}{*}{ D3 } & & & & \\
\hline & $\mathrm{D}$ & $3.285,75$ & 718,15 & $4.003,90$ & & $\mathrm{D}$ & $3.116,14$ & 931,27 & $4.047,41$ \\
\hline & C & $3.378,75$ & 655,55 & $4.034,30$ & & C & $3.461,34$ & 779,47 & $4.240,81$ \\
\hline & B & $3.462,55$ & 494,04 & $3.956,59$ & & & & & \\
\hline & A & $3.800,44$ & 500,48 & $4.300,92$ & & A & $3.964,62$ & 777,64 & $4.742,26$ \\
\hline \multirow{5}{*}{$\mathrm{C} 2$} & $E$ & $3.091,58$ & 820,40 & $3.911,98$ & \multirow{5}{*}{ E1 } & & & & \\
\hline & $\mathrm{D}$ & $3.252,58$ & 711,70 & $3.964,28$ & & D & $2.779,45$ & 1232,29 & $4.011,74$ \\
\hline & $\mathrm{C}$ & $3.447,30$ & 663,19 & $4.110,49$ & & $\mathrm{C}$ & $3.133,25$ & 947,46 & $4.080,71$ \\
\hline & $B$ & $3.521,60$ & 491,70 & $4.013,30$ & & $B$ & $3.272,52$ & 777,07 & $4.049,59$ \\
\hline & A & $3.944,57$ & 647,04 & $4.591,61$ & & A & $3.640,56$ & 986,27 & $4.626,83$ \\
\hline
\end{tabular}


elementos constructivos como cimentación y estructura, 25 años para el reemplazamiento de diferentes elementos de la vivienda como pavimentos, alicatados, equipamientos de cocina, etc. y 15 años para instalaciones, como las calderas $(15,16,17)$. La vida útil del edificio adoptada, de 100 años, se basa en la Orden ECO/805/2003, de 27 de marzo, sobre normas de valoración de bienes inmuebles y de determinados derechos para ciertas finalidades financieras, al ser la que se adopta en usos residenciales. El método de amortización a utilizar va a ser siempre el lineal con valor residual 0 . La amortización se obtiene a partir del coste de la inversión, con los incrementos mencionados, dividido por 100, 25 o 15 años, según la vida útil del elemento considerado (18). El coste de amortización, expresado en €/año, será así comparable al coste de la energía.

Se calcula el coste de amortización ya que es importante considerar la vida útil del edificio, al tratarse de un bien de consumo con un largo periodo de uso. La construcción y la demolición son operaciones que requieren de elevadas cantidades de energía, aunque poco significativas si las comparamos con el uso del edificio (19).

También se han calculado los costes del consumo de energía, considerando la suma del consumo por calefacción, refrigeración y ACS. Las tarifas de electricidad y gas natural son las vigentes en el año 2010 y se obtienen del Boletín Oficial del Estado de 31 de Diciembre de 2009. El coste de los pellets en la solución de biomasa incluye costes de portes.

Con respecto a la amortización, en la Tabla 8 se puede observar que, cuanto mejor es la calificación energética más alta es la amortización, tal y como cabría esperar.

Con respecto a los costes de consumo, cabría esperar que fueran menores a mejor calificación energética. Sin embargo, no siempre se obtiene ese resultado. Los casos en que esto no ocurre están señalados en negrita en la Tabla 8. Cuando se utiliza la caldera de biomasa (calificación A), las emisiones de carbono $\left(\mathrm{kg} \cdot \mathrm{CO}_{2} / \mathrm{m}^{2} \cdot \mathrm{año}\right)$ se reducen mejorando la calificación energética, pero el consumo anual de energía ( $\mathrm{kW} \cdot \mathrm{h} / \mathrm{m}^{2}$.año) y su coste no se reducen (Tablas 7 y 8 ), al no utilizar Calener VYP este indicador cuando otorga la calificación energética al edificio.

En la Tabla 8 el coste total se calcula como la suma de la amortización más el coste de consumo. Para promover la eficiencia energética en edificios entre los usuarios, el coste total debería ser menor cuanto mayor fuera la calificación energética. Sin embargo, se observa que esto no ocurre en los casos se- ñalados en negrita, ya que la reducción en el consumo no compensa la amortización. Esto se podría haber alcanzado mediante el uso del indicador $\mathrm{kW} \cdot \mathrm{h} / \mathrm{m}^{2} \cdot$ año en el programa, junto al de las emisiones de $\mathrm{CO}_{2}$.

\section{CONCLUSIONES}

En este artículo se estudia una herramienta disponible para los profesionales, Calener VYP, que tiene como objetivo la certificación energética de edificios, con la intención de promover la mejora de la eficiencia energética en los mismos. Su aplicación en el mundo profesional tendrá como consecuencia la obtención de edificios certificados energéticamente. Por ello, es interesante analizar el procedimiento seguido por la herramienta para otorgar la calificación energética y cuáles son las implicaciones a nivel económico, ya que sólo se garantizará el éxito de la mejora de la eficiencia energética en el caso de que las medidas aplicadas resulten rentables.

Se ha comprobado que la herramienta oficial para obtener la calificación energética de nuevos edificios en España, Calener VYP, utiliza un indicador, emisiones anuales de $\mathrm{CO}_{2}$, que posibilita asegurar que cuanto mejor es la calificación energética, menores son las emisiones producidas por el edificio. Sin embargo, el indicador consumo anual de energía primaria, también calculado por el software, no es tenido en cuenta al otorgar la calificación energética al edificio simulado. Como consecuencia, mejores calificaciones energéticas no implican necesariamente menor coste de consumo energético o coste total. La distorsión introducida por el hecho de no utilizar el indicador de consumo anual de energía primaria se hace muy evidente cuando se selecciona la caldera de biomasa, la cual el programa considera neutral a efectos de emisiones anuales de $\mathrm{CO}_{2}$, y por tanto, la calificación obtenida es independiente de la demanda energética de calefacción del edificio.

Se puede concluir que, utilizando Calener VYP, no se alcanzan mejoras costo-efectivas en la totalidad de la calificación energética del edificio. Como consecuencia, de acuerdo a los resultados obtenidos en el caso de estudio, el ahorro de costes no puede ser utilizado como argumento para promover la eficiencia energética entre usuarios y propietarios. Dicha costo-efectividad podría lograrse simplemente incluyendo el indicador consumo energético a la hora de otorgar la calificación energética. Asimismo, la caldera de biomasa dejaría de ser un elemento distorsionante porque aunque su valor fuera nulo en lo relativo al indicador de emisiones de $\mathrm{CO}_{2}$, el consumo energético de la misma estaría determinando la calificación a obtener. 
Asimismo, se han estudiado las medidas que contribuyen a la reducción de emisiones al menor coste de inversión posible y que permiten pasar de una calificación energética dada a otra mejor, dentro de la tipología determinada de viviendas adosadas. Salvando las medidas de diseño y de tipo pasivo, que sin coste añadido permiten mejorar la eficiencia energética, se han analizado combinaciones entre diferentes soluciones constructivas y sistemas de instalaciones. Se concluye que, entre las estudiadas, las que permiten mayor reducción de emisiones por euro de inversión tienen el orden siguiente: incrementar el espesor de la capa de aislamiento en la envolvente térmica del edificio, el uso de radiadores en calefacción, la mejora del comportamiento térmico de los vidrios, el uso de calderas no eléctricas, los vidrios bajo emisivos, la carpintería exterior de madera y las calderas de biomasa.

Por último, según los resultados obtenidos, se puede concluir que sólo se alcanzan las mejores calificaciones A por medio de la caldera de biomasa, ya que las emisiones debidas a calefacción se consideran nulas con este combustible. Se plantea en este punto la conveniencia de que futuras versiones de los programas que se empleen para certificar energéticamente edificios, permitan implementar medidas diferentes al uso de biomasa para obtener la calificación A, como el suelo radiante, los cerramientos ventilados, y las energías geotérmica y fotovoltaica.

\section{AGRADECIMIENTOS}

Agradecemos a Jara Teba, de IBERDROLA Inmobiliaria, por proporcionarnos el proyecto utilizado como caso de estudio y su ayuda en todas las aclaraciones que posteriormente fuimos necesitando respecto al mismo. Agradecemos también a Edurne Zubiri y Miguel Ángel Pascual, de Natural Climate Systems S.A. (MIYABI), por las discusiones acerca del uso de Calener-VYP v1.0.

\section{BIBLIOGRAFÍA}

(1) Concerted Action EPBD (2008): "Implementation of the Energy Performance of Buildings Directive", Country reports 2008, Brussels: Directorate General for Energy and Transport, European Commission.

(2) Pérez-Lombarda, L.; Ortiz, J.; Poutb, C.: "A review on buildings energy consumption information", Energy and Buildings, vol. 40, n 3 (2008), pp. 394-398.

(3) Directive 2010/31/UE of the European Parliament and of the Council of 19 May 2010 on the energy performance of buildings: Official Journal of the European Union 18.06.2010. ISSN 17252555. doi:10.3000/17252555.L_2010.153.eng.

(4) Míguez, J. L.; Porteiro, J.; López-González, V; Vicuña, J. E.; Murillo, S.; Morán, J. C.; Granada, E.: "Review of the energy rating of dwellings in the European Union as a mechanism for sustainable energy", Renewable and Sustainable Energy Reviews, vol. 10, no 1 (2006), pp. 24-45.

(5) García-Casals, X.: "Analysis of building energy regulation and certification in Europe: Their role, limitations and differences", Energy and Buildings, vol. 38, nº 5 (2006), pp. 381-392.

(6) AICIA (2009a): Escala de calificación energética. Edificios de nueva construcción, Madrid: Instituto para la Diversificación y Ahorro de la Energía, Ministerio de Industria, Turismo y Comercio.

(7) IDAE (2007): Calener-VYP v1.0. Manual de usuario, Madrid: Ministerio de Vivienda e Instituto para la Diversificación y Ahorro de la Energía (IDAE).

(8) AICIA (2009b): Condiciones de aceptación de procedimientos alternativos a Lider y Calener, Madrid: Instituto para la Diversificación y Ahorro de la Energía, Ministerio de Industria, Turismo y Comercio.

(9) Alavedra, P.; Domínguez, J.; Engràcia, G.; Serra, J.: "La construcción sostenible. El estado de la cuestión", Informes de la Construcción, vol. 49, nº 451 (1997), pp. 41-45.

(10) Universidad Politécnica de Madrid. Departamento de Construcción y Vías Rurales (2009): Evaluación de los costes constructivos y consumos energéticos derivados de la calificación energética de viviendas. Precost \& E. Fase1

(11) UNE-EN ISO 6946:1997/A1:2005: Building components and building elements - Thermal resistance and thermal transmittance - Calculation method (ISO/DIS 6946:2005).

(12) Ruá, M. J.; López-Mesa, B.; Vives, L; Civera, V.: “Environmental Advantage of Back-Ventilated Cladding Façades in Comparison with Brick Cavity Walls in Residential Buildings", Advances in Energy Research, vol. 8, chapter 10, ed. Morena V. Acosta, Nova Science Publishers, Inc. 2012.

(13) Uzsilaityte, L.; Martinaitis. V.: "Search for optimal solution of public building renovation in terms of life cy-

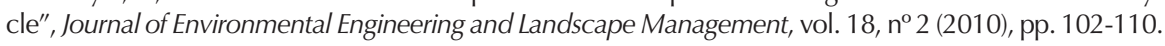

(14) Bertrán-Moreno, A.: Las mediciones en las obras adaptadas al CTE, ed. 4ª ., Jorge Lorigi S.L., Granada, 2009.

(15) Davies, H.; Wyatt, D.: "Appropiate use or method for durability and service life prediction", Building Research and Information, vol. 32, nº 6 (2004), pp. 552-553. doi: 10 1080/0961321042000291938.

(16) Liska, R. W.: Means Facilities Maintenance Standards, R.S. Means Company, Inc. Construction Publishers \& Consultants, 2000.

(17) Rudbeck, C. "Service life of building envelope components: making it operational in economical assessment", Construction and Building Materials, vol. 16, nº 2 (2002), pp. 83-89.

(18) Llano Elcid, A.: Valoraciones Inmobiliarias: fundamentos teóricos y manual práctico, ed. 7ª , Llano Realtors, S.L., Vizcaya, 2007.

(19) Lecuona-Neumann, A.; Izquierdo-Millán, M.; Rodríguez-Aumente, P. A.: "Investigación e impacto ambiental de los edificios. La energía", Informes de la Construcción, vol. 57, nº 498 (2005), pp. 47-61. 